\title{
The influence of late preterm birth on infant body composition at term corrected age
}

\author{
A. Doolan ${ }^{1}$, A. O’Higgins ${ }^{3}$, C. O'Connor ${ }^{3}$, M. M. Kennelly ${ }^{3}$, M. J. Turner ${ }^{3}$, E. Roche ${ }^{2}$ and \\ M. Sheridan-Pereira ${ }^{1}$ \\ ${ }^{1}$ Department of Paediatrics, Trinity College Dublin, Coombe Women and Infants University Hospital, Dublin 8, \\ ${ }^{2}$ Department of Paediatrics, Trinity College Dublin, National Childrens Hospital, Tallaght, Dublin 24 and ${ }^{3}$ UCD Centre \\ for Human Reproduction, Coombe Women and Infants University Hospital, Dublin 8, Ireland
}

Preterm birth and birth weight have lifelong implications for an individual's metabolic health ${ }^{(1)}$. Preterm infants born before 32 completed weeks of pregnancy have altered body composition when they reach term ( $>37$ weeks gestation) ${ }^{(2,3)}$. As there is a dearth of body composition data on late preterm (32-37 weeks gestation) infants, we hypothesised that they would similarly have altered body composition at term.

Infants who were born between 32-37 weeks gestation and were admitted to the Neonatal Unit in the Coombe Women and Infants University Hospital (CWIUH) were eligible for recruitment. Their body composition was measured at term corrected age. Term infants (37-40 weeks gestation) who as participants in maternal-infant research studies had their body composition measured prior to hospital discharge, served as study controls. Both groups of infants had anthropometric measurements and body composition analysis performed (using the PEA POD Infant Body Composition System, Cosmed, Rome, Italy). The study was approved by the research ethics committee in the CWIUH and informed consent was obtained from the parents of all infants. Data were analysed using IBM SPSS Statistics 19.

53 late preterm infants reaching term and 134 term control infants had body composition analysis performed. Preterm infants had a mean gestation of 34.6 weeks (SD 1.17) and birth weight $2.21 \mathrm{~kg}$ (SD 0.4), while term infants had a mean gestation of 39.7 (SD 1.35 ) and birth weight of $3.54 \mathrm{~kg}$ (SD 0.5). Preterm infants weighed less at term, with reduced fat free mass and increased fat mass when compared with term born infants (Table 1).

Table 1.

\begin{tabular}{lccc}
\hline & Preterm (SD) & Term (SD) & $p$ value \\
\hline Gestation (weeks) & $40.1(0.9)$ & $39.9(1.3)$ & 0.2 \\
Weight (kg) & $\mathbf{3 . 2 2}(\mathbf{0 . 5})$ & $\mathbf{3 . 4 1}(\mathbf{0 . 5})$ & $\mathbf{0 . 0 2}$ \\
\%Fat mass (\%) & $\mathbf{1 4 . 2}(\mathbf{3 . 9})$ & $\mathbf{1 1 . 1}(\mathbf{4 . 7})$ & $<.001$ \\
\%Fat free mass (\%) & $\mathbf{8 5 . 7}(\mathbf{3 . 9})$ & $\mathbf{8 8 . 8}(\mathbf{4 . 7})$ & $<\mathbf{0 . 0 0 1}$ \\
Fat mass $(\mathbf{k g})$ & $\mathbf{0 . 4 7}(\mathbf{0 . 1 8})$ & $\mathbf{0 . 3 9}(\mathbf{0 . 2})$ & $\mathbf{0 . 0 2}$ \\
Fat free mass $(\mathbf{k g})$ & $\mathbf{2 . 7 4}(\mathbf{0 . 3 3})$ & $\mathbf{3 . 0 2}(\mathbf{0 . 3 8})$ & $<\mathbf{0 . 0 0 1}$ \\
\hline
\end{tabular}

Though late preterm infants at term are lighter than control term born infants, they have more adipose tissue. This demonstrable difference at term in their fat and fat free mass proportions raises concern for a negative metabolic impact and resultant lifelong health disparities. Follow-up body composition analyses on these children during their childhood years are warranted to determine whether this difference in body composition persists beyond term.

1. Euser AM, Finken MJ, Keijzer-Veen MG et al. (2005) Am J Clin Nutr 81, 480-7.

2. Cooke RJ, Griffin I. (2009) Acta Paeditr 98, 1269-73.

3. Roggero P, Gianni ML, Amato O et al. (2007) JPGN 45, S159-S162. 